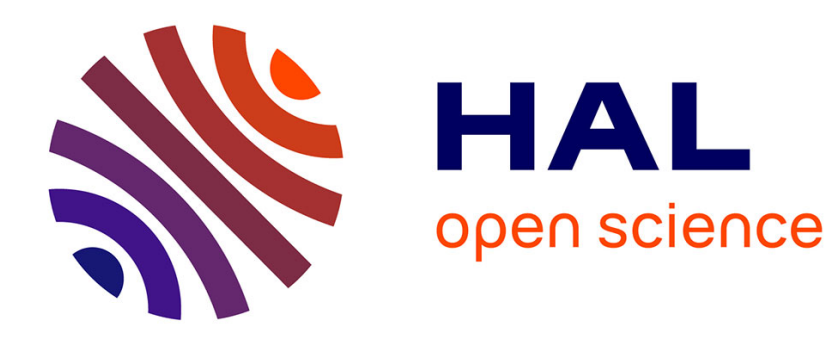

\title{
Phénomènes pseudo-photographiques
}

P. Villard

\section{To cite this version:}

P. Villard. Phénomènes pseudo-photographiques. J. Phys. Theor. Appl., 1908, 7 (1), pp.506-520. 10.1051/jphystap:019080070050601 . jpa-00241334

\section{HAL Id: jpa-00241334 https://hal.science/jpa-00241334}

Submitted on 1 Jan 1908

HAL is a multi-disciplinary open access archive for the deposit and dissemination of scientific research documents, whether they are published or not. The documents may come from teaching and research institutions in France or abroad, or from public or private research centers.
L'archive ouverte pluridisciplinaire HAL, est destinée au dépôt et à la diffusion de documents scientifiques de niveau recherche, publiés ou non, émanant des établissements d'enseignement et de recherche français ou étrangers, des laboratoires publics ou privés. 


\section{PHÉNOMĖNES PSEUDO-PHOTOGRAPHIQUES (1);}

Par M. P. VILLARD.

En 1860, de La Blanchère écrivait:

"Par l'addition d'un peu de solution argentifère au liquide pyrogallique, une image négative faible vire instantanément au positif et se développe ensuite normalement dans cet état. " Le même auteur ajoute que, conformément à une observation faite par Schouwaloff

(1) Communication faite à la Société française de Physique : Séance du ว janvier 1906. 
(Art du Photographe), on remarque, entre la partie positive et la partie négative de l'image, une auréole blanche plus claire que les deux.

Le $\mathrm{D}^{\mathrm{r}} \mathrm{A}$. Guébhard a obtenu le mème résultat en traitant par un révélateur étendu une plaque au gélatino-bromure d'argent, faiblement exposée pour avoir, comme plus haut, un négatif faible, et laissant séjourner la plaque dans le bain pendant un temps considérable; le bromure d'argent non attaqué pouvait ainsi se dissoudre en quantité notable dans le sulfite de soude du révélateur, ce qui revenait au même que d'ajouter au réducteur une solution argentifère.

Dans ce genre d'expériences il se produit, outre l'auréole claire signalée par Schouvaloff, ce que les photographes appellent une inversion. Celte expression, universellement adoptée, a le défaut de donner une idée fausse des phénomènes. On fait involontairement la comparaison avec ce qui se passe parfois dans une machine de Holtz, et on est tenté de se représenter une image comme un objet indivisible, dont toutes les parties sont liées, et qui passe du négatif au positif, ou inversement, par une sorte de mouvernent de bascule, les blancs devenant des noirs et réciproquement.

Ce qui se passe est moins mystérieux : une image photographique est un ensemble de particules métalliques dont l'objectif a réglé la répartition, et chacune d'elles ne peut agir autour d'elle que dans un rayon comparable à ses dimensions; ce qui se passe à une extrémité d'une plaque $13 \times 18$ n'a aucune influence sur ce qui peut se produire à l'autre extrémité.

Quand une inversion se produit au cours d'un développement, c'est que, pour une raison ou une autre, les blancs du négatif obtenu d'abord se remplissent d'un dépôt dont l'opacité arrive à dépasser celle des teintes les plus fortes du négatif, ces dernières continuant d'ailleurs à noircir de plus en plus. Dans aucun cas l'inversion ne comporte l'éclaircissement des noirs du négatif, moins encore le transfert des particules d'argent d'une région à une autre par redissolution suivie d'une nouvelle précipitation. Un bain réducteur ne saurait redissoudre l'argent qu'il vient de réduire. Il peut seulement arriver qu'à la longue le sulfite de soude du révélateur dissolve le bromure d'argent non réduit: ce fixage éclaircit nécessairement l'épreuve et il en résulte une erreur d'interprétation qu'on doit éviter en ne comparant les épreuves qu'après fixage de celles qui contiennent encore du bromure d'argent. Une action extrêmement pro- 
longée, au cours de laquelle l'oxydation complète du réducteur ne laisse subsister que le pouvoir dissolvant du sulfite de soude, peut, il est vrai, affaiblir légèrement l'image; mais cet affaiblissement ne porte pas uniquement sur la partie négative de l'épreuve. On vérifie par exemple aisément qu'un séjour prolongé dans l'hyposulfite de soude éclaircit sensiblement un cliché. L'effet est d'autant plus marqué qu'il s'agit de préparations à grain plus fin : il est très appréciable au bout de cinq à six heures avec les papiers au gélatinobromure d'argent, et le ton de l'épreuve vire au violacé (ton chaud) par diminution du diamètre des particules d'argent.

Dans le cas de la solarisation, c'est la lumière qui détermine l'inversion. La vitesse de développement (poids d'argent réduit par unités de surface et de temps) est d'autant plus grande, mais d'autant plus vite décroissante, que l'exposition a été plus forte. Les régions surexposées noircissent les premières, mais sont ensuite rattrapées et dépassées par les autres, l'ensemble s'assombrissant d'ailleurs de plus en plus.

Il en est tout autrement dans les expériences citées au début de cet article.

On constate d'abord, et tous les auteurs sont d'accord sur ce point, que ce sont les clichés très sous-exposés qui s’inversent le plus facilement par l'addition d'une liqueur argentifère au révélateur, ou par l'immersion prolongée dans un bain capable de dissoudre du bromure d'argent. Quand se forme l'image positive qui va imposer son signe à l'épreuve, c'est par les plages les moins exposées qu'elle débute, et de préférence par celles qui n'ont reçu aucune lumière (régions protégées par le châssis).

Si l'expérience est faite en traitant par un révélateur dilué $\left(^{4}\right)$ un cliché au gélatino-bromure d'argent, on observe que l'inversion continue à se produire longtemps après que tout le bromure d'argent a été dissous, et que sa disparition a entraîné celle de l'impression lumineuse localisée par l'objectif. La formation de l'image positive ne peut donc être attribuée quà la présence du bain d'argenture formé par le bromure d'argent dissous dans le révélateur. Cette manière de voir est confirmée par les remarques suivantes: l'inversion est notablement accélérée par l'addition, dans le même bain,

(1) Par exemple, une solution de métoquinone dans le sulfite de soude (1. gramme de métoquinone et 10 grammes de sulfite par litre). Ce révélateur, exempt d'alcali libre, ne tend pas à décoller la gélatine. 
de plusieurs plaques, impressionnées ou non; par la présence d'une trace d'hyposulfite de soude; par l'augmentation de la teneur en sulfite. Elle devient très rapide si on verse dans la cuve quelques gouttes d'une solution toute préparée de chlorure ou bromure d'argent dans le sulfite de soude.

On observe, d'autre part, que la transformation de l'épreuve s'accompagne de phénomènes accessoires qui suivent une marche exactement parallèle : les parois de la cuve se recouvrent d'un dépôt d'argent plus ou moins sulfuré, le liquide se trouble et laisse déposer de l'argent, du sulfure d'argent et même du soufre. Tout ce qui accélère ou accentue l'inversion accroît en mème temps l'intensité de ces effets et la rapidité de leur production. C'est donc bien la présence d'un bain d'argenture qui détermine la formation d'une image positive; il est remarquable que non seulement ce bain ne renforce que très peu le négatif d'abord obtenu, mais que l'intensité du positif complémentaire continue à augmenter après la disparition complète du bromure d'argent de la plaque. Il est, par suite, certain que les noirs du positif ne sont pas formés de la même manière que ceux du négatif, mais sont dus à une véritable argenture de la gélatine.

Le dépôt qui constitue cette image déposée ainsi en second lieu est très différent de celui qui forme les clichés ordinaires par réduction des grains de bromure d'argent sur place. Il est formé de grains beaucoup plus fins et, dans certains cas, paraît n'être pas constitué par de l'argent réduit. Avec les plaques Jouğla par exemple, si on arrête l'expérience dès que le positif a franchement dépassé en intensité le négatif initial, il suffit de tremper l'épreuve dans l'hyposulfite de soude pour voir disparaître tout le dépôt complémentaire, et il ne subsiste que le négatif ordinaire à peine voilé.

En présence de ces faits, on est conduit à admettre avec MM. Lumière qu'on est en présence d'un voile dichrö̈que simplement exagéré. Ce voile, jaunâtre par transmission et bleuâtre par réflexion, est également formé par un dépôt à grains extrêmement fins, et il se produit pendant le fixage, c'est-à-dire au moment où la gélatine contient à la fois un réducteur et de l'argent dissous (hyposulfite double d'argent et de sodium), c'est-à-dire un bain d'argenture. La lumière facilite la formation de ce voile, mais elle n'est pas nécessaire et, avec certains révélateurs surtout (pyrocatéchine, métoquinone), on obtient un voile intense en se servant d'un hyposulfite déjà usagé, c'est-à-dire chargé d'argent et de révélateur, ou même simplement en évitant de 
laver le cliché avant le fixage; on réussit encore mieux en remettant le négatif dans le révélateur au sortir du bain de fixage ( $\left.{ }^{(}\right)$.

Or ce voile présente une propriété extrêmement remarquable d'autant plus facile à observer que sa teinte le distingue franchement de l'image proprement dite : très intense dans les régions tout à fait transparentes de l'épreuve, beaucoup plus faible dans les parties déjà teintées, nul dans les grands noirs de l'épreuve, il constitue, en jaune, le complément du négatif, c'est-à-dire une image positive. En raison de cette propriété, il est particulièrement intense dans les clichés à grande transparence, c'est-à-dire sous-exposés. Cette dernière condition est justement celle qui convient le mieux pour obtenir qu'une image vire du négatif au positif.

Ce qui se passe dans l'expérience de de La Blanchère, ou avec les plaques au gélatino-bromure d'argent abandonnées dans un bain faible, consiste donc en ceci :

Les particules de chlorure ou de bromure d'argent impressionnées sont d'abord réduites et constituent le négatif ordinaire. On ajoute alors au bain une dose notable d'azotate d'argent, ou on laisse la plaque dans le révélateur qui a déjà dissous du bromure d'argent et continue à s'en charger de plus en plus. Dans les deux cas, on a un cliché dans un bain d'argenture qui tend à déposer du métal partout, sur les parois de la cuve aussi bien que sur l'épreuve, et il en résulte d'abord un léger accroissement des grains d'argent de l'image, c'està-dire un renforcement de celle-ci. Mais ce renforcement s'arrête bientôt, et les particules non seulement cessent d'attirer l'argent en voie de réduction, mais s'opposent à sa précipitation dans les régions qu'elles occupent dans le collodion ou la gélatine (noirs du négatif). Le métal va donc se déposer surtout dans les plages inoccupées, et en quantité d'autant plus grande que ces plages seront plus parfaitement exemptes de tout grain d'argent; de là une réussite plus cer-. taine avec les clichés sous-exposés, présentant de grandes transparences. Les noirs du négatif ne permettront aucune précipitation, les demi-teintes donneront un effet intermédiaire. Le dépôt d'argent produit par le bain sera donc complémentaire de l'image donnée par le développement ordinaire, ce sera un positif ; son intensité pourra

(1) De La Blanchère avait déjà signalé la formation d'un voile rougeâtre pendant le fixage, et il avait vérifié que ce voile se produit très bien en versant une solution d'acide pyrogallique sur un négatif qu'on vient de fixer au cyanure de potassium. 
être très supérieure à celle du négatif dont il comble les vides, car la grosseur des particules déposées peut devenir la même que pour ce négatif, mais leur nombre n'est pas limité par celui des granules de l'émulsion; il dépendra seulement du nombre des germes capables d'attirer l'argent, poussières ou précipités salins contenus dans la gélatine, ou peut-être particules colloïdales de celle-ci.

On verra done se former d'abord un voile dichroïque pusitif, qui peu à peu se renforcera par accroissement de grosseur des particules qui le composent et semblera subir, comme l'indique de Ia Blanchère, un véritable développement, à la fin duquel le positif obtenu l'emportera notablement comme opacité sur le négatif initial; l'ensemble de l'épreuve sera par suite positif, les blancs étant toutefois constitués par les noirs du négatif. Ces noirs seront d'autant plus clairs que l'exposition aura été moindre et pourront, par contraste, paraître relativement limpides.

Si cette manière de voir est exacte, s'il s'agit non de phénomènes photochimiques, mais d'attractions et de répulsions réciproques entre petites particules, dues peut-être à leur signe électrique, on peut se débarrasser de toute la partie photographique de l'expérience et employer par exemple des clichés fixés, lavés et séchés, et rendus ainsi insensibles à la lumière; on doit devenir tout à fait maìtre des résultats en perfectionnant les bains d'argenture; enfin des effets toutà fait semblables pourrontêtre obtenus avec des substances autres que l'argent. L'expérience vérifie complètement ces prévisions et montre que ce genre de phénomènes est d'une extrême généralité.

On prend, par exemple, un négatif stéréoscopique extrêmement faible, fixé et lavé depuis longtemps, on imprègne l'une des images d'une dissolution concentrée de bromure d'argent dans le sulfite de soude, et on la traite par un bain de métoquinone et sulfite de soude; l'autre image sert de témoin (1). On voit aussitôt se former un voile dichroïque qui se renforce rapidement, dépasse bientôt en intensité le négatif, et l'inversion est complète en quelques instants ( $f g$. 1). On voit que les noirs du négatif, jouant maintenant le rôle de blancs, sont légèrement renforcés. On peut également immerger le négatif dans un bain de métoquinone et sulfite de soude contenant un peu de chlorure ou de bromure d'argent dissous dans du sulfite de soude.

(1) Dans toutes ces expériences, j'ai utilisé constamment les plaques Lumière afin de réduire le nombre des paramètres variables, parmi lesquels intervient la perméabilité de la gélatine. 
Suivant la concentration, l'expérience dure à volonté dix minutes ou plusieurs jours ( $\left.{ }^{1}\right)$.

Le ton de l'épreuve est violacé, la teinte variant un peu suivant qu'on prend du chlorure ou du bromure d'argent.

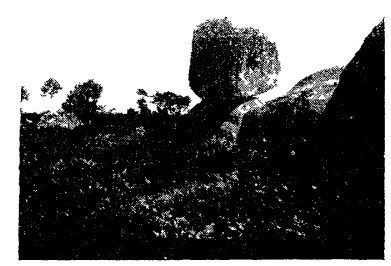

FIG. 1.

On réussit également très bien en traitant le négatif par une dissolution d'azotate d'argent, puis par un révélateur. L'inversion est alors extrêmement rapide et le dépôt d'argent est gris bleuâtre.

Dans toutes ces expériences, il faut avoir soin de prendre des négatifs très limpides; ils peuvent d'ailleurs être très faibles, ce qui montre à quel point les particules d'argent préexistantes s'opposent au dépôt ultérieur : le moindre voile gênerait beaucoup la formation de ce dépôt (à moins bien entendu que ce ne soit le voile dichroïque).

On réussit également bien avec une solution de sulfite d'argent dans le sulfite de soude, ou de chlorure ou bromure d'argent dans l'hyposulfite, ce dernier cas correspondant précisément aux conditions dans lesquelles se forme d'ordinaire le voile dichroïque.

Le bain d'argenture peut être remplacé par d'autres solutions métalliques. On peut par exemple prendre un cliché semblable à celui de la fig. 1, ou plus simplement un négatif consistant en un cercle blanc bien limpide entouré d'un fond gris (ombre d'une pièce de monnaie), et l'imprégner, avant ou après fixage, d'une solution de chlorure d'or contenant une trace d'acide oxalique. On traite ensuite par l'oxalate neutre de potasse (température $20^{\circ}$ environ). Le négatif est d'abord viré par substitution, puis le cercle blanc se remplit

(1) Quand on mélange la solution argentifère au réducteur, on ne peut employer des liqueurs aussi concentrées que dans le cas précédent; on obtiendrait une réduction en masse de l'argent dissous avant que la gelatine n'ait été pénétrée par le réactif. 
d'un dépôt intense d'or bleu par transparence et fortement miroitant par réflexion sur le côté gélatine : c'est de la dorure localisée.

La $f g .2$ montre le résultat d'une expérience de ce genre; le négatif (ombre d'une roue d'engrenage) a été traité après fixage comme il vient d'être dit, puis éclairé par une lampe et photographié dans la direction de la réflexion régulière. On voit que le dépôt miroitant est strictement limité au contour du négatif.

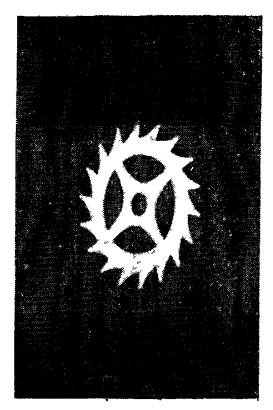

FIG. 2.

On peut supprimer le traitement par l'oxalate de potasse; l'acide oxalique suffit, à la condition d'attendre plus longtemps.

Le rôle des germes est facile à mettre en évidence avec les dépôts d'or. Un faible voile d'oxalate de chaux, obtenu par un lavage à l'eau de la Vanne suivi d'un traitement par l'acide oxalique, facilite sensiblement la formation du dépôt.

Le sulfate ferreux concentré. maintenu entre $+20^{\circ}$ et $+22^{\circ}$, convient également comme réducteur et donne de l'or bleu verdâtre. La réduction est extrêmement rapide.

On peut répéter la même expérience en remplaçant le chlorure d'or par le chloropalladite de potassium qu'on verse sur le cliché avec un peu de formiate de soude. On obtient un positif brun formant un très beau miroir de palladium.

Le chloroplatinite de potassium convient également; il suffit, après imprégnation du cliché par le sel, de réduire par l'oxalate ferreux tiède $\left(3 \check{5}^{\circ}\right)$. En une ou deux opérations on obtient un brillant miroir de platine occupant toute la région transparente du négatif. L'ensemble est positif par transparence.

J. de Phys., $4^{\circ}$ série, t. VII. (Juillet 1908.) 
Dans tous ces exemples, les noirs du négatif sont nécessairement virés et ne peuvent être enlevés après l'opération. On peut les faire disparaître dans les cas suivants :

Le négatif fixé et lavé est traité par le permanganate de potasse (au besoin légèrement acidulé). L'argent disparaît et à sa place se produit un dépôt brun. Ce nouveau négatif, formé par des grains d'hydrate manganique, se comporte vis-à-vis de l'or comme le cliché d'argent. On le traite par une solution de chlorure d'or additionnée d'acide acétique et on réduit par le sulfate ferreux tiède. L'or qui se dépose respecte les régions occupées par l'hydrate manganique, d'autant plus parfaitement qu'elles sont plus intenses; on traite ensuite l'épreuve par l'acide oxalique ou chlorhydrique ou le bisulfite de soude pour enlever le manganèse, et il reste un positif d'or dont les blancs sont cette fois presque purs (inversion vraie).

L'intérêt de cette expérience résulte surtout de la suppression complète de l'argent, c'est-à-dire de la matière provenant d'une opération photographique. On voit en même temps qu'une substance autre que ce métal peut localiser par exclusion la précipitation d'un métal en voie de réduction.

Au point de vue de la beauté des images, le meilleur résultat s'obtient en transformant d'abord en ferricyanure ammoniacal l'argent d'un cliché intense et à grandes transparences. On le traite pour cela par le cyanure rouge à $20 / 0$ additionné d'une trace d'ammoniaque. Le ferricyanure d'argent ammoniacal oppose un obstacle presque absolu à la formation d'un dépôt d'or. Si donc on traite le négatif en ferricyanure par ldu chlorure d'or('), puis par l'oxalate de potasseà saturation et tiède, l'or, d'abord violet, puis rose, se dépose en premier lieu dans les grandes transparences du cliché; en réitérant l'opération, les demi-teintes sont envahies à leur tour, et, en dissolvant ensuite le sel d'argent par l'iodure de potassium( $\left(^{2}\right)$, on obtient un très beau positif, semblable à un vitrail, dont les blancs sont absolument parfaits ( $f y .3$ ).

Le ton obtenu est très différent de celui que donne ordinairement I'oxalate employé comme réducteur. Cette différence parait tenir au tannage que le cyanure fait subir à la gélatine; cette circonstance a d'ailleurs l'avantage de permettre l'emploi d'un bain réducteur tiède.

(1) Solution à $10 / 0$ sans acide oxalique, à cause du sel ammoniacal.

$\left(^{2}\right)$ On pourrait employer l'hyposulfite de soude. 
On peut enfin prendre comme point de départ une image n'ayant pas une origine photographique :

Une lame de gélatine sur verre (plaque au bromure d'argent fixée et lavée sans impression ni développement) est plongée quelques
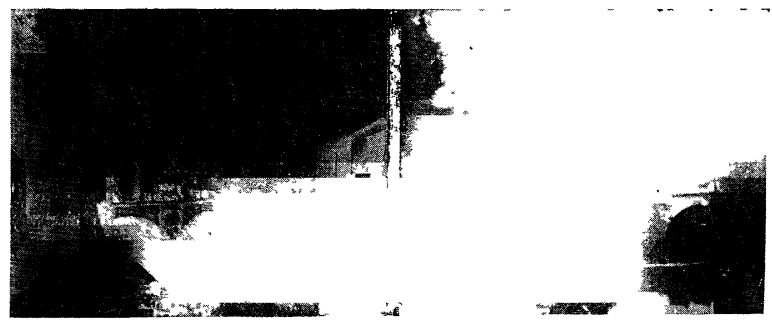

Fig. 3. - Transformation d'un négatif ordinaire en positif à blancs purs. (Epreuve stéréoscopique dont la moitié droite a été transformée, l'autre moitié servant de témoin).

instants dans une solution de chromate de potasse; un dessin quelconque est ensuite tracé sur la gélatine avec un pinceau chargé de nitrate de plomb; on obtient ainsi un précipité colloïdal transparent de chromate de plomb, localisé à volonté. Après lavage, on plonge successivement la plaque dans de l'azotate de baryte, puis dans du sulfate de soude. On observe invariablement que le précipité de sulfate de baryte est complètement exclu des plages déjà occupées par le chromate de plomb, et celles-ci se détachent, par transparence, en jaune clair, sur fond presque opaque ( $f g .4)$.

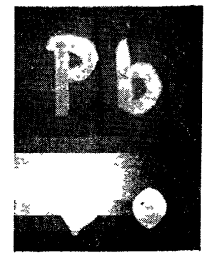

FIG. 4.

Cette expérience montre clairement que la lumière ne joue aucun rôle dans ces phénomènes.

Tous les précipités ne possèdent pas la propriété d'exclure ainsi un autre précipité. Dans certains cas, la répulsion est remplacée par une attraction. Un dépôt d'or rose, par exemple, attire l'argent en voie de 
précipitation. On s'en assure aisément en déposant sur de la gélatine une goutte de chlorure d'or qu'on réduit par l'oxalate de potasse; si on fait agir ensuite l'un des bains d'argenture indiqués plus haut, tout l'argent se dépose sur les particules d'or, et l'opacité de la tache d'or devient presque complète.

Certains précipités argentiques possèdent la même propriété attractive, par exemple les granules qui constituent les images de noircissement direct sur gélatino-chlorure ou gélatino-bromure d'argent, ou sur papiers dits pour noircissement direct.

FIG. 5.

L'épreuve reproduite $(f g . \breve{5})$ a été obtenue de cette manière : une plaque Perron au gélatino-chlorure d'argent à grain très fin (émulsion pour tons chauds) a été exposée environ vingt minutes à la lumière sous un négatif. L'image positive très faible ainsi obtenue a été recouverte d'une solution de chlorure d'or à $1 / 20 / 0$ additionnée d'une trace d'acide oxalique. L'or réduit s'est déposé presque uniquement sur les particules constituant l'image, et ce développement physique a donné une épreuve intense d'or vert. Cette expérience réussit d'autant mieux que le grain est plus fin, sans doute parce qu'il y a en pareil cas un plus grand nombre de points d'appel, de germes, par unité de surface.

Le rôle des germes et celui de la grosseur des particules peuvent être mis en évidence d'une manière frappante par l'expérience suivante :

On traite un vieux cliché par la liqueur de Farmer (hyposulfite de soude et ferricyanure de potassium); tout l'argent se dissout, mais à la place qu'il occupait subsiste soit une déformation de la gélatine, soit une trace de dépôt calcaire provenant des eaux de lavage et ayant cristallisé sur les grains de métal. 
Après lavage, et même séchage, on traite cette gélatine par une solution de chlorure d'argent dans le sulfite de soude et le révélateur à la métoquinone. Aussitôt l'imuge détruite se reforme, d'abord à grain très fin ; elle se renforce peu à peu, noircit par suite du grossissement des grains et revient à sa valeur primitive. Si on essaye d'aller au delà, c'est alors le voile dichroïque qui apparaît, puis l'image complémentaire qui aboutit à l'inversion. On peut détruire à nouveau l'image réapparue, laver et recommencer. La trace laissée par cette image dans la gélatine est presque indélébile.

\section{SILHOUETTAGE.}

Dans nombre de journaux illustrés qui publient des photogravures, les nécessités de la mise en pages obligent souvent à faire empiéter un sujet sur le sujet adjacent. La tête d'un personnage est, par exemple, enclavée dans le premier plan d'un paysage placé audessus. Pour que la partie enclavée se détache, on l'entoure d'un trait blanc qui en suit exactement la silhouette. Ce dédoublement, ici artificiel, des contours d'un objet, peut définir ce qu'il convient d'entendre par le mot silhouettage.

On observe un phénomène absolument identique dans un certain nombre d'expériences photographiques, et ce phénomène a été signalé pour la première fois par Schouwaloff, précisément dans les cas d'inversion par addition de liqueur argentique à un révélateur.

Ce liséré blanc se produit en effet invariablement dans toutes les expériences décrites au chapitre précédent $\left(^{1}\right)$; il est la conséquence nécessaire de l'incompatibilité de site de deux dépôts différents. Une plage occupée par des grains d'argent n'est en effet jainais à bords rigoureusement nets, quelle que soit la manière dont elle s'obtient; sa limite réelle est donc un peu au delà de son contour apparent : or c'est la limite réelle qui définit le domaine du dépôt formé ultérieurement. Le liséré clair représenterait alors la largeur de la pénombre invisible. Cette manière de voir est d'accord avec le fait qu'en produisant un dépôt intense on arrive à combler presque entièrement la bande claire en question. Dans le cas où le signe électrique des précipités interviendrait, une répulsion à distance se comprendrait d'ailleurs aisément.

(1) Sauf peut-être pour l'or réduit par l'hydrosulfite de soude. 
Il est évident que ce silhouettage ne peut être que blanc(1), et qu“il est toujours pris sur le dépôt formé en second lieu. Ses bords, toujours très nets, sont définis par une fine ligne sombre qui renforce le contour des plages séparées par la bande claire. Cette ligne a une autre origine que le silhouettage, c'est celle qu'on observe sur le bord d'une tache. On l'obtient, par exemple, en déposant sur de la gélatine une goutte de chlorure d'or ou d'azotate d'argent qu'on réduit ensuite. Cette ligne, qui renforce le contour de la tache, n'a évidemment aucune analogie avec le phénomène dont il s'agit; un trait de force n'est pas un silhouettage, il accentue un contour, il ne le double pas.

Les opérations photographiques ordinaires consistant à obtenir des dépôts de particules dans du collodion ou de la gélatine permet- . tront naturellement d'obtenir le silhouettage. La condition nécessaire à réaliser sera de produire, par un moyen quelconque, deux dépôts différents occupant des plages contiguës. Cette condition sera en général suffisante. Voici quelques-uns des procédés qu'on peut employer.

On éclaire pendant un instant, aussi court que l'on veut, une plaque antihalo sur laquelle est posée une cache de forme quelconque $\left({ }^{2}\right)$. On développe, on lave, puis on expose toute la plaque à la lumière, en tenant compte de la moindre sensibilité du bromure mouillé. On a ainsi défini deux régions exactement contiguës et dont chacune n'a été exposée à la lumière qu'une fois, car elles ont été protégées l'une par la cache pendant la première pose, l'autre pendant la seconde par l'argent réduit constituant un véritable cliché appliqué sur le bromure sous-jacent. Ces deux expositions peuvent être à peu près quelconques, le résultat n'en est pas modifié. On remet ensuite la plaque dans le révélateur: la plage primitivement réservée se développe, et, à tout instant de l'opération, on observe qu'elle reste séparée de l'autre par un silhouettage clair ; mais on remarque aussi que, dans la partie exposée à sec, l'argent réduit est noir, tandis qu'il est brun et à grain plus fin, dans la région impressionnée après mouillage.

(1) Si on pouvait produire un troisième dépôt exclu par les deux premiers, il tendrait évidemment à remplir le silhouettage et à le noircir, mais il serait luimême silhouetté de blanc de part et d'autre, de telle sorte que l'énoncé subsisterait.

(2) Pour éviter la pénombre optique, on emploie la lumière parallèle. 
Le résultat est encore meilleur en faisant la seconde exposition pendant que la plaque est dans le révélateur, mais alors le ton de la plage développée ainsi est violacé, ou même rose (avec le diamidophénol étendu). Suivant la durée de cette deuxième opération, l'ensemble de l'épreuve sera positif ou négatif, cela dépendra des valeurs relatives des plages complémentaires, mais le silhouettage sera le même dans les deux cas; il résulte de ce que les particules réduites dans les deux phases de l'expérience sont différentes comme grain (elles diffèrent également au point de vue chimique).

La $f g .6$ représente indifféremment les divers silhouettages obtenus avec l'or, le platine, le palladium, l'argent, ou par voie photographique. Dans la première épreuve, le second dépôt commence à rem-
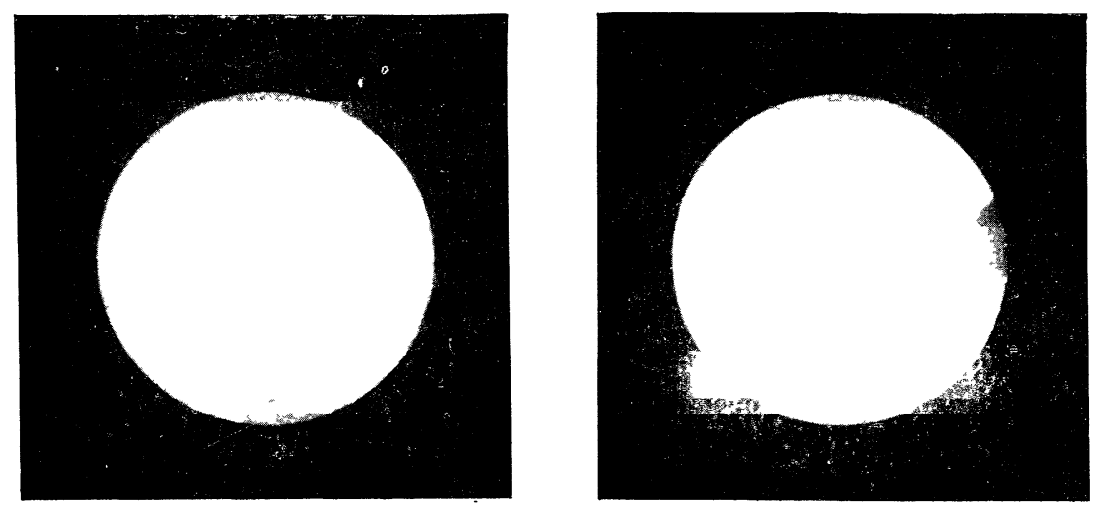

FIG. 6.

plir les blancs du négatif; dans la seconde, il l'emporte en intensité sur le fond, et l'ensemble est un positif. L'image a été grossie 4 fois.

Enfin on peut se contenter d'une exposition unique : on éclaire uniformément toute la surface d'une plaque, puis l'on dépose sur elle deux ou trois gouttes de révélateur à la pyrocatéchine. Au bout de deux secondes on lave à grande eau, puis on développe à l'hydroquinone. La place occupée par chaque goutte est légèrement plus noire que le fond environnant, et elle s'entoure d'une auréole claire. Si on inverse l'ordre des révélateurs, le phénomène disparaît presque complètement. 
Inversement, on peut supprimer toute opération photographique. La fig. 7 montre, grossi 4 fois, le silhouettage obtenu en prenant de la gélatine imbibée de chromate de potasse et déposant à sa surface

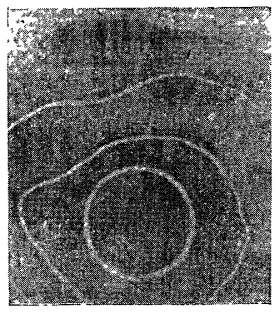

FIG. 7.

une goutte d'une dissolution d'azotate de plomb qu'on étale par saccades en ajoutant brusquement de nouvelles quantités de solution. A chaque opération il se produit une zone nouvelle de chromate de plomb, mais dans des conditions un peu différentes au double point de vue de la composition du liquide et du gonflement de la gélatine. Il en résulte que les précipités colloïdaux successifs sont séparés les uns des autres par une bande claire.

Cette dernière expérience et celle réalisée avec le sulfate de baryte démontrent que ce genre de phénomènes n'a rien de commun avec les actions chimiques de la lumière. 